\title{
eRHIC Beam Scrubbing
}

\author{
S.Y. Zhang
}

\section{Collider-Accelerator Department Brookhaven National Laboratory Upton, NY 11973}

\author{
U.S. Department of Energy \\ Office of Science, Office of Nuclear Physics
}

Notice: This document has been authorized by employees of Brookhaven Science Associates, LLC under Contract No. DE-SC0012704 with the U.S. Department of Energy. The United States Government retains a nonexclusive, paid-up, irrevocable, world-wide license to publish or reproduce the published form of this document, or allow others to do so, for United States Government purposes. 


\section{DISCLAIMER}

This report was prepared as an account of work sponsored by an agency of the United States Government. Neither the United States Government nor any agency thereof, nor any of their employees, nor any of their contractors, subcontractors, or their employees, makes any warranty, express or implied, or assumes any legal liability or responsibility for the accuracy, completeness, or any third party's use or the results of such use of any information, apparatus, product, or process disclosed, or represents that its use would not infringe privately owned rights. Reference herein to any specific commercial product, process, or service by trade name, trademark, manufacturer, or otherwise, does not necessarily constitute or imply its endorsement, recommendation, or favoring by the United States Government or any agency thereof or its contractors or subcontractors. The views and opinions of authors expressed herein do not necessarily state or reflect those of the United States Government or any agency thereof. 


\title{
eRHIC Beam Scrubbing
}

\author{
S.Y. Zhang *
}

June 22. 2017

\begin{abstract}
We propose using beam scrubbing to mitigate the electron cloud effect in the eRHIC. The bunch number is adjusted below the heat load limit, then it increases with the reduced secondary electron yield resulted from the beam scrubbing, up to the design bunch number. Since the electron density threshold of beam instability is lower at the injection, a preliminary injection scrubbing should go first, where large chromaticity can be used to keep the beam in the ring for scrubbing. After that, the beam can be ramped to full energy, allowing physics scrubbing. Simulations demonstrated that with beam scrubbing in a reasonable period of time, the eRHIC baseline design is feasible.
\end{abstract}

\section{Secondary electron yield}

One of the most important parameter in the electron cloud is the secondary electron yield (SEY), which determines the production of secondary electrons due to the impinging electrons pushed to wall by the passing beam bunches.

The SEY consists of two parts. The first is the "true secondary yield" and the second is the reflected electrons.

The "true" secondary yield can be described by 3 parameters [1] along with the electron energy $E$, which are the peak electron yield $\delta_{\max }$, the electron energy at the peak yield, $E_{\max }$, and a fitting parameter $s$,

$$
\delta_{\text {true }}(E)=\delta_{\max } \frac{s E / E_{\max }}{s-1+\left(E / E_{\max }\right)^{s}}
$$

The reflected electron yield can be described by 2 parameters [2], which are $R_{0}$ and $E_{0}$,

$$
\delta_{r e f l}(E)=R_{0}\left(\frac{\sqrt{E}-\sqrt{E+E_{0}}}{\sqrt{E}+\sqrt{E+E_{0}}}\right)^{2}
$$

*Acknowledgement: I would like to thank M. Blaskiewicz for many helpful discussions 

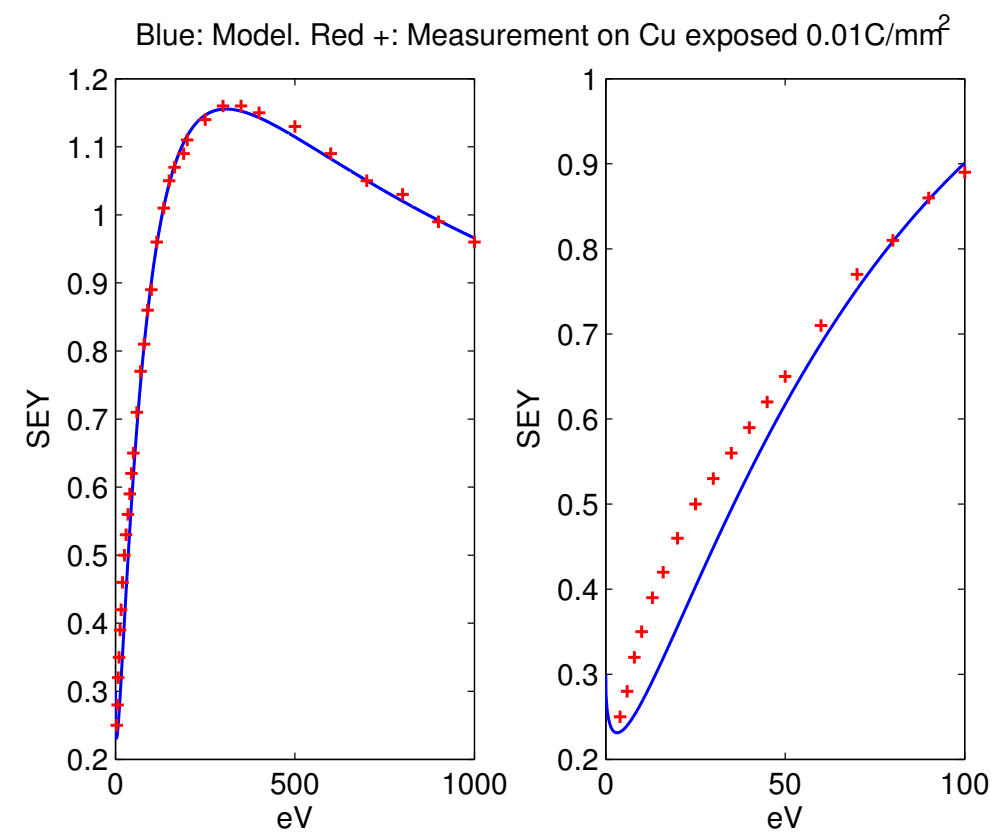

Figure 1: SEY model with $E_{\max }=315 \mathrm{eV}, R_{0}=0.3, E_{0}=225 \mathrm{eV}, \mathrm{s}=1.35, \delta_{\max }=1.15$ is compared with the measurement of the $\mathrm{Cu}$ surface exposed by a dose of $0.01 \mathrm{C} / \mathrm{mm}^{2}$

Extensive experimental studies have been performed on the copper $(\mathrm{Cu})$ surface, which is similar to the $\mathrm{Cu}$ coating surface proposed for eRHIC arc chamber. For the SEY model, the parameters of the true and reflected yields are chosen based on the fitting of the experimental measurement, up to $1000 \mathrm{eV}$.

The parameters of the SEY used for eRHIC electron cloud simulations are $E_{\max }=315 \mathrm{eV}$, $R_{0}=0.3, E_{0}=225 \mathrm{eV}$, and $s=1.35$.

In Figure 1, this SEY model is compared with the experimental measurement on $\mathrm{Cu}$ surface with $\delta_{\max }=1.15$, reported in [3].

The usual peak SEY for the received $\mathrm{Cu}$ surface is $\delta_{\max }=2.1$, which will be reduced with the given electron dose. For example, in Figure 1, the $\mathrm{Cu}$ surface in the measurement was exposed by a dose of $0.01 \mathrm{C} / \mathrm{mm}^{2}$, i.e., 0.01 Coulomb per $\mathrm{mm}^{2}$, and the peak SEY is reduced to $\delta_{\max }=1.15$.

\section{Electron dose}

It is useful to deposit electron dose on a surface in order to reduce SEY. For an accelerator, the passing beam induced electron cloud would have this effect, and it is called beam scrubbing.

It has been demonstrated that higher impinging electron energy would have higher SEY reduction rate for given dose.

In [4], a systematic measurement is presented for electron bombardment on $\mathrm{Cu}$ surface with 
different impinging electron doses and energies. The measurement is performed for the normal incidents, which have perpendicular impinging angle of the electron on the target.

Large Hadron Collider (LHC) has used the beam scrubbing as the major tool to reduce the chamber surface SEY in order to accommodate the bunches with smaller bunch spacing, which produce heavier electron cloud for given SEY.

From the electron density produced by the electron cloud per unit length, usually a meter, $d n_{e} / d s$, the dose can be estimated,

$$
D_{s}=f_{0} T \frac{e M d n_{e} / d s}{a}
$$

where $f_{0}$ is the revolution frequency, $T$ is the total time, $M$ is the bunch number, and $a$ timed the unit length is the area the electron dose deposited. If the dose applied evenly on the wall of a round chamber with a radius of $b$, then $a=2 \pi b$.

With eRHIC, for example, given $d n_{e} / d s=10^{9} / \mathrm{m}$, for $f_{0}=78 \times 10^{3}, M=330, b=35 \mathrm{~mm}$, then in 1 hour, the dose received on chamber surface is $D_{s}=0.067 \mathrm{mC} / \mathrm{mm}^{2}$.

Note that this calculation is assuming that the electron dose spreads evenly on the chamber surface. If not, then $2 \pi b$ should be replaced by the relevant area of the scrubbing.

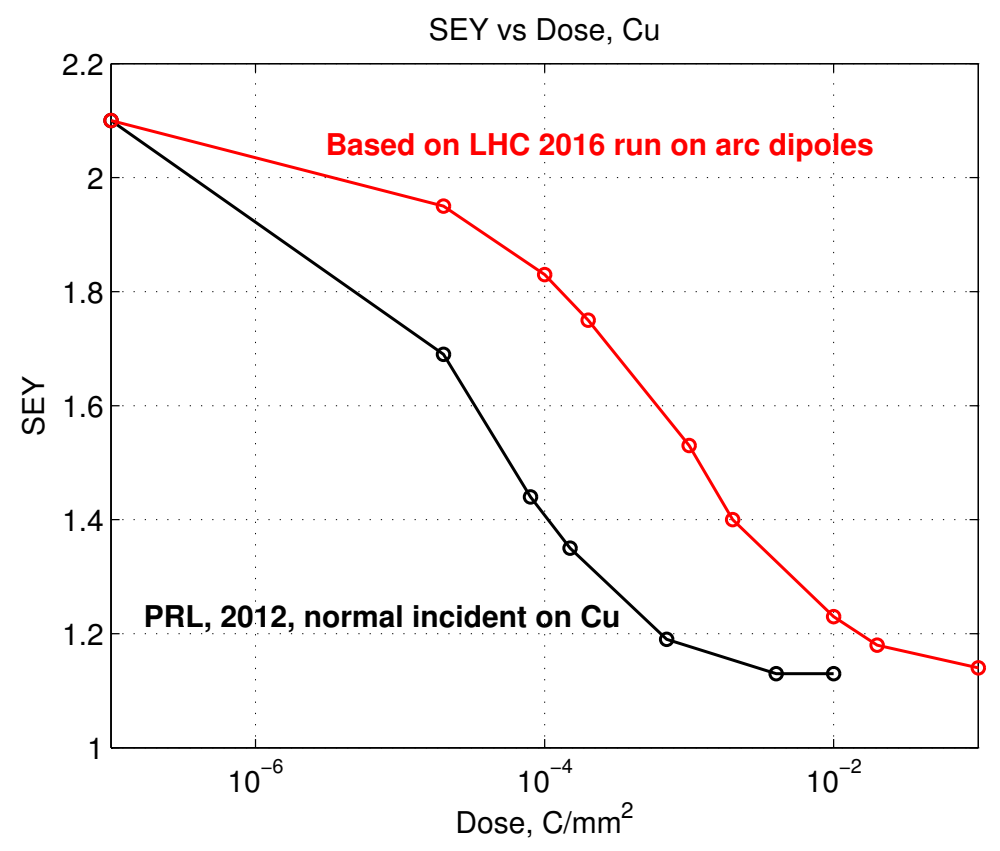

Figure 2: Electron dose and SEY measured in Lab for the normal incidents at the impinging electron energy of $200 \mathrm{eV}$, and the one modified according to the LHC 2016 run experience. The latter is used as the guidance for eRHIC beam scrubbing.

The verified relation of the dose and SEY reduction in the machine beam scrubbing is not the same as the lab measurement. In [5], it is reported that, after the entire LHC run 2016, 
"Estimated electron dose in the arc dipole during 2016 is at least 20 times larger than the dose needed in the lab", where the lab measurement is referred to that from [4].

In Figure 2, the dose and SEY relation presented in [4] is at the impinging electron energy of $200 \mathrm{eV}$, which is comparable with the average impinging electron energy for eRHIC. Also, with the experience presented in LHC operations, the more practical dose and SEY reduction relation is presented, which will be used as the eRHIC beam scrubbing guidance.

\section{Heat load}

Among the negative effects of electron cloud, the heat load poses a hard restriction, which is limited by the cryogenic capacity. The LHC commissioning and operations have demonstrated that the heat load is always of concern, and the cryogenic limit is never being far away.

The heat load can be estimated if the electron density and the impinging electron energy are known, as

$$
\frac{d P}{d s}=k \frac{d n_{e}}{d s} \frac{\langle\triangle E\rangle}{t_{b s}}
$$

where $d P / d s$ is the heat load, $\langle\triangle E\rangle$ is the average energy the electrons hitting the wall, $t_{b s}$ is the bunch spacing in time, and $k$ represents other effects relevant to the average heat load, such as the beam aborting gap, etc.

Taking $k=0.9, d n_{e} / d s=10^{9} / \mathrm{m},\langle\triangle E\rangle=200 \mathrm{eV}$, then with $t_{b s}=35 \mathrm{~ns}$ for $M=330$ at eRHIC, the heat load is $d P / d s=0.82 \mathrm{~W} / \mathrm{m}$.

The heat load contribution at the eRHIC arc is dominated by the dipoles, which consist about $90 \%$ of the arc. On the other hand, the contribution of the quadrupole is not negligible. The overall heat load, which is limited by the cryogenic capacity of $1 \mathrm{~W} / \mathrm{m}$ at the eRHIC, needs to consider both dipoles and quadrupoles.

To obtain the heat load for a given SEY, based on the eRHIC baseline design [6] of the bunch intensity, emittance, bunch spacing, bunch length, etc, simulations have been performed.

PyECLOUD [7], which is developed based on ECLOUD at CERN, and used for the LHC commissioning and operation (also for SPS and PS) is adequate for the eRHIC electron cloud effect study.

In Figure 3, for the eRHIC dipole at the store, the heat load, the average impinging electron energy, and the total energy of the impact electrons on the wall are shown by the simulations with respect to different SEY.

The received $\mathrm{Cu}$ typically has SEY with $\delta_{\max }=2.1$. With the eRHIC baseline design of $N_{b}=1.1 \times 10^{11}$ protons, the heat load of total 330 bunches at store is clearly above the cryogenic capacity of $1 W / m$ at $\delta_{\max }=2.1$, even only considering the contribution of the dipoles.

For eRHIC, it is necessary to reduce the SEY, which can be accomplished by the large dose of the electron bombardment provided by the beam scrubbing.

As Figure 3 shows, if the SEY reduced to $\delta_{\max }=1.2$, the heat load would be below $1 \mathrm{~W} / \mathrm{m}$. Since the quadrupoles' contribution is less than the dipoles, this also demonstrate that the 
PyECLOUD Simulation, eRHIC Baseline Design

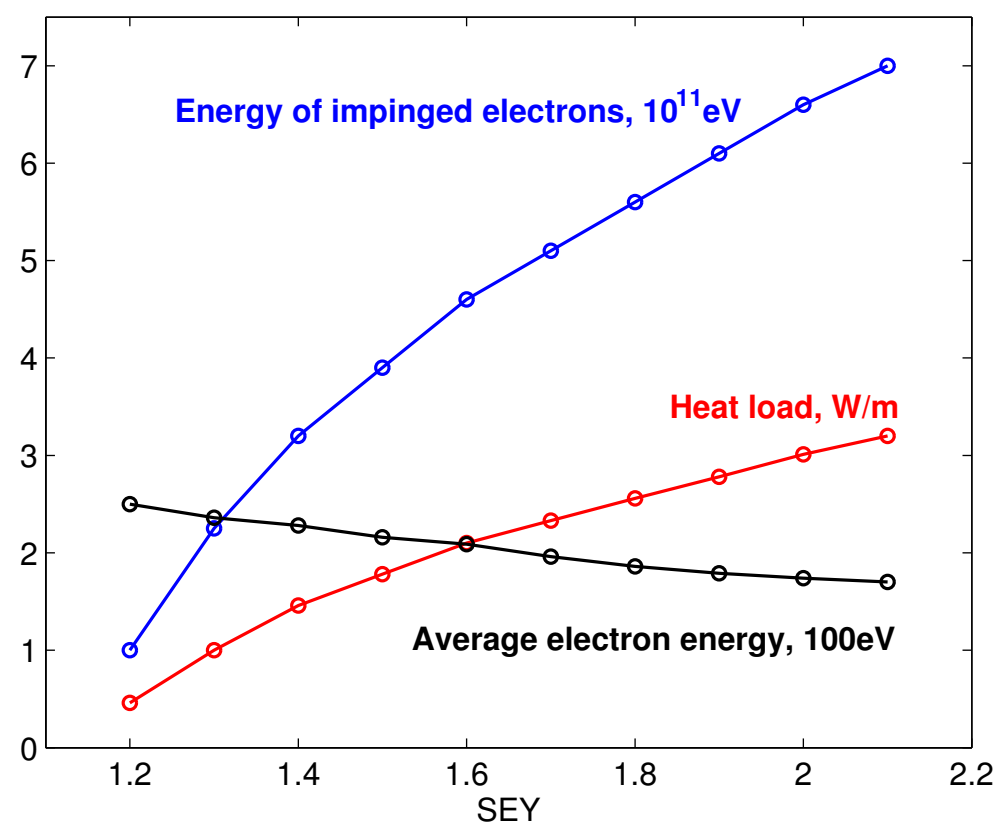

Figure 3: Simulation results using PyECLOUD for eRHIC baseline design of dipoles for the heat load at the store, for different SEY. Shown also the average impinging electron energy, and the total energy of the impact electrons on the wall.

eRHIC baseline design, considering the heat load from both dipoles and quadrupoles, can be reached with the SEY reductions.

\section{Beam scrubbing}

Consider beam scrubbing, the heat load limit of $1 \mathrm{~W} / \mathrm{m}$ has to be observed. With the heat load much less than that, the electron dose rate will be lower. Therefore, for an efficient beam scrubbing, the best way is to use the beams with the heat load just below $1 \mathrm{~W} / \mathrm{m}$.

For the dipole area, the electron cloud buildup are strongly influenced by externally applied magnetic fields, and the electrons move along with 2 or 3 vertical stripes. As the simulations demonstrated, the impinged area on the wall are determined mainly by the bunch intensity. Therefore, it is important to keep the bunch intensity the same as that in physics runs, in order to scrub the relevant area in the wall.

The strategy of the beam scrubbing is, therefore, to use the bunches with the design bunch intensity and bunch spacing, then adjust the bunch number so the heat load is just below $1 W / m$. With the rising accumulated dose in the scrubbing, the chamber SEY will reduce, as shown in Figure 2.

For the reduced SEY, more bunches can be applied for the scrubbing, keeping the heat load 
unchanged. This procedure is repeated, until the design bunch number, say for eRHIC baseline design $M=330$, is reached.

In fact, before reaching the full bunch number, a parasitic physics run with the experimental data taking may take place. This is called the physics scrubbing.

The beam scrubbings keep going on, even with the full physics run. After all, the less the electron cloud effect, the better for machine runs.

For beam scrubbing, the bunch number can be determined with the heat load setting at $1 W / m$, by

$$
M=\frac{h t_{b s}}{k d n_{e} / d s\langle\triangle E\rangle}
$$

where $h$ is the harmonic number, for eRHIC baseline design, $h=360$.

If one takes $k=0.9$, with the initial electron density of $d n_{e} / d s=4 \times 10^{9} / \mathrm{m},\langle\triangle E\rangle=200 \mathrm{eV}$, then we get $M=109$, which means that 109 bunches can be injected with the heat load not above $1 W / m$.

For the dipole area, the simulation shows the total impinging area is about $a \times 1000=$ $40 \times 10^{3} \mathrm{~mm}^{2}$ in a meter long chamber.

Using Equation 3, the accumulated dose in 1 hour for the case shown above with $M=109$ is $D_{s}=0.49 \mathrm{mC} / \mathrm{mm}^{2}$.

From Figure 2, with this dose, the SEY should be reduced from $\delta_{\max }=2.1$ to a little higher than $\delta_{\max }=1.6$.

In Figure 4, the entire scrubbing process is illustrated by the reduction of SEY from $\delta_{\max }=$ 2.1 to $\delta_{\max }=1.2$. The bunch number and the needed time for beam scrubbing are shown for each step of the SEY reduction.

Some comments follow:

1. During the entire beam scrubbing, the heat load is kept constant at $1 \mathrm{~W} / \mathrm{m}$, and only in the last step, the full bunch number produces heat load lower than $1 W / m$, at the reduced $\mathrm{SEY} \delta_{\max }=1.2$.

2. The bunch number is increased according to the reduced SEY, from $M=79$ bunches at the beginning of the scrubbing to the full bunch number of $M=330$.

3. The scrubbing time is increased in each step of the SEY reduction. The later reduction approaching SEY of $\delta_{\max }=1.2$ takes longer time, which is agreeable with the dose and SEY relation shown in Figure 2.

The SEY reduction steps is only for illustration, and in scrubbing, more steps may be needed, especially in the later scrubbing.

In practice, the cryogenic flow can be monitored for estimating the dose received by the beam scrubbing, which is to decide the bunch numbers to use. 


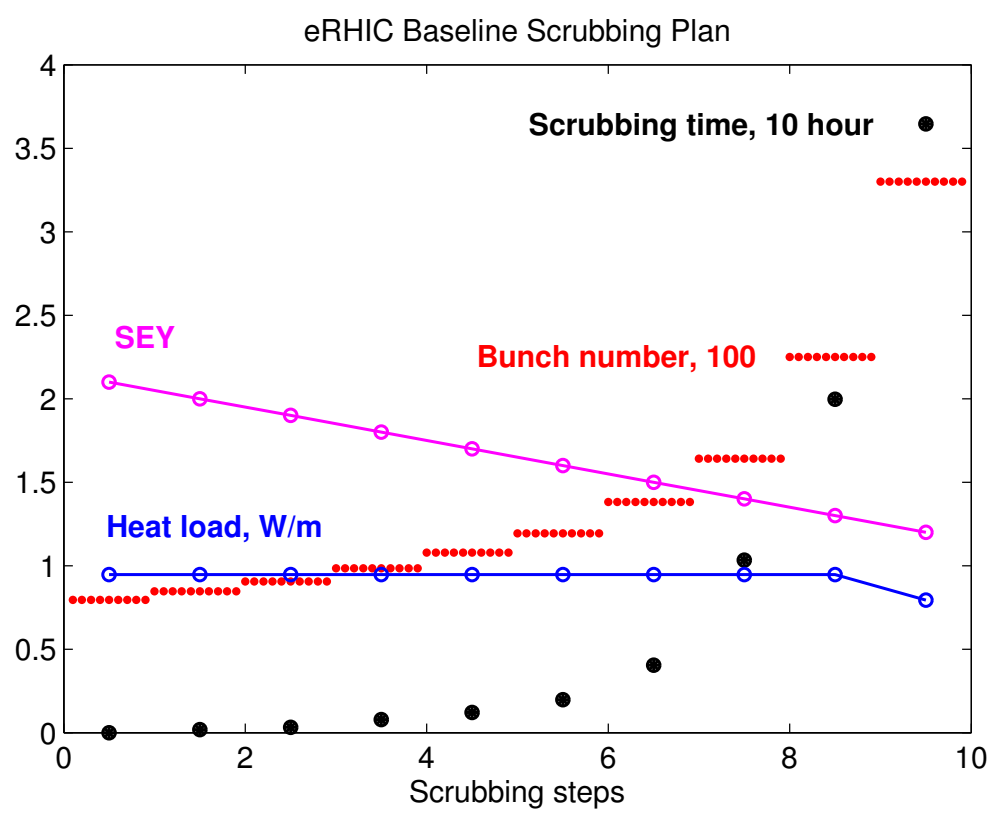

Figure 4: Illustration of a beam scrubbing for eRHIC. To adjust the bunch number to keep the heat load at $1 W / m$, the chamber SEY is reduced, which allows more bunches for scrubbing, until the full bunch number is reached. The time of scrubbing is increased as approaching SEY of $\delta_{\max }=1.2$. Both dipole and quadrupole contributions in the heat load are included in the scrubbing plan.

\section{$5 \quad$ Electron cloud in beam scrubbing}

For the efficient beam scrubbing, one needs to push the highest electron production to deposit highest dose on the wall.

The electron cloud effect, therefore, will not be negligible, and the beam instability and the emittance growth/blow-up will cause beam loss and to shorten the beam lifetime, which will affect, or even prevent, the beam scrubbing.

The most important parameter for the effect of electron cloud on beam is the total electrons in the machine chamber. Usually the average electron density in ring is used to evaluate the electron cloud effect on beam.

In the proposed beam scrubbing, by changing the bunch number and keep the constant heat load, the average electron density in ring in fact will not change much from the start of the beam scrubbing to early physics run.

With the heat load limit of $d P / d s=1 W / m$, from an average electron energy, the electron density per unit length in arcs is approximately determined from Equation 4.

For example, in eRHIC, using $k=0.9$, with $t_{b s}=35 \mathrm{~ns}$, the average electron energy of $\langle\triangle E\rangle=250 \mathrm{eV}$ implies the electron density of $d n_{e} / d s=0.972 \times 10^{9} / \mathrm{m}$.

From the simulations performed on eRHIC baseline design, which produces the beam scrubbing shown in Figure 4, the average electron density with varying bunch number during the 
beam scrubbing is $d n_{e} / d s=0.978 \times 10^{9} / \mathrm{m}$, and the average electron energy is $\langle\triangle E\rangle=252 \mathrm{eV}$. This shows that the estimate of the electron density from the heat load limit and the average electron energy is acceptable.

We use this approach to estimate the electron density in ring from the start of the beam scrubbing to early physics run.

Consider only the electron density in the arcs (taking average electron density over the ring it will be a little lower), then the average electron density in ring with the eRHIC baseline design is shown in Table 1.

The LHC parameter and the estimated electron density in ring are also shown for comparison, where $\langle\triangle E\rangle=350 \mathrm{eV}$ is assumed.

\begin{tabular}{|c|c|c|c|c|c|c|c|c|c|c|}
\hline & $k$ & $t_{b s}$ & $d P / d s$ & $\langle E\rangle$ & $d n_{e} / d s$ & $b_{x} / b_{y}$ & $\ell$ & $n_{e, \text { total }}$ & $V$ & $n_{e} / V$ \\
\hline & & $n s$ & $W / m$ & $e V$ & $10^{9} / m$ & $c m$ & $m$ & $10^{12}$ & $m^{3}$ & $10^{12} / m^{3}$ \\
\hline & & & & & & arc & $80 \%$ of $C$ & & arc & \\
\hline eRHIC & 0.9 & 35 & 1 & 250 & 0.972 & $3.5 / 3.5$ & 3,000 & 2.92 & 11.5 & 0.254 \\
\hline LHC & 0.9 & 25 & 2.2 & 350 & 1.091 & $2.3 / 1.8$ & 22,000 & 24.0 & 28.6 & 0.839 \\
\hline
\end{tabular}

Table 1. Average electron density for eRHIC and LHC at the beam scrubbing and early runs, where $b_{x}$ and $b_{y}$ are the chamber half height in the arcs.

The eRHIC electron density of $0.254 \times 10^{12} / \mathrm{m}^{3}$ is about 7 times of the RHIC has experienced in run 2005, where the beam transverse emittance growth of $50 \%$ has been observed, with no clearly observed beam instabilities.

To estimate the effect of the electron density on beam, usual approach is to find the electron density threshold of the single bunch TMCI (transverse mode coupling instability) or strong head-tail instability [8].

With the synchrotron motion, the electron density threshold $\rho_{e t h}$ of the head-tail instability is shown in,

$$
\rho_{e t h}=\frac{2 \gamma Q_{s}}{\pi r_{p} C\langle\beta\rangle}
$$

where $Q_{s}$ is the synchrotron tune, $C$ is the machine circumference, and $\langle\beta\rangle$ is the average beta function.

In Table 2, the electron density threshold for head-tail instability of eRHIC and LHC at injection and store are presented.

For eRHIC, the RF parameters at the store are given in [6], but that at the injection, i.e., the harmonic number, the RF voltage, are the assumed, which is close to the RHIC proton runs.

By comparing the electron density of $0.254 \times 10^{12} / \mathrm{m}^{3}$ and $0.839 \times 10^{12} / \mathrm{m}^{3}$ for eRHIC and LHC, respectively, with the electron density threshold shown in Table 2 , both machines would have beam instabilities at the injection, but not at the store.

The reported observations at LHC are in general agreeable, as shown in $[7,9]$. 


\begin{tabular}{|c|c|c|c|c|}
\hline & \multicolumn{2}{|c|}{ eRHIC baseline } & \multicolumn{2}{c|}{ LHC } \\
\hline & Injection & Store & Injection & Store \\
\hline Relativistic gamma, $\gamma$ & 25.4 & 271 & 480 & 7461 \\
\hline RF frequency, $f_{r f}, M H z$ & 28.1 & 394.1 & 400.8 & 400.8 \\
\hline RF Voltage, $V_{r f}, M V$ & 0.3 & 13.8 & 8 & 16 \\
\hline Longit. emittance, $A_{\ell}, e V s$ & 0.8 & 0.8 & 1.0 & 2.5 \\
\hline rms bunch length, $\sigma_{s}, \mathrm{~cm}$ & 59.6 & 9.82 & 11.25 & 7.5 \\
\hline Synch. frequency, $f_{S}, H z$ & 40.8 & 716 & 63.6 & 22.9 \\
\hline Synch. tune, $Q_{s}, 10^{-3}$ & 0.52 & 9.2 & 5.7 & 2.0 \\
\hline Average beta function, $\langle\beta\rangle, m$ & 22 & 22 & 80 & 80 \\
\hline E-density threshold, $\rho_{e t h}, 10^{12} / m^{3}$ & 0.065 & 12.2 & 0.52 & 2.86 \\
\hline
\end{tabular}

Table 2. eRHIC and LHC parameters relevant to the electron density threshold of head-tail instability. eRHIC injection RF voltage and harmonic number are the assumed. The electron density threshold at the injection is much lower than that at the store.

\section{$6 \quad$ Injection and physics scrubbing}

The electron density in eRHIC ring presented in Table $1, n_{e} / V=0.254 \times 10^{12} / \mathrm{m}^{3}$, is larger than the head-tail instability electron density threshold of $0.065 \times 10^{12} / \mathrm{m}^{3}$ at the injection, shown in Table 2. This indicates that there will be electron cloud induced beam instability and transverse emittance growth/blow-up at the injection and ramp.

A preliminary beam scrubbing at the injection, therefore, is necessary.

To inject a beam into eRHIC below the head-tail instability electron density threshold of $0.065 \times 10^{12} / \mathrm{m}^{3}$ is a possible choice, however, then the induced electron density would be too low to have effective beam scrubbing.

Consider Equation 6, the synchrotron tune can be raised to enhance the electron density threshold to allow for stronger beam, for example with higher RF voltage. This is an option, with the larger beam momentum spread $d p / p$.

Another way to mitigate the beam instability is using larger chromaticity to raise the electron density threshold, where the increased chromatic tune plays a similar role of the synchrotron tune as a damping force.

In fact, LHC has used the larger chromaticity to accommodate new beams with smaller bunch spacing, and hence stronger electron cloud. For example, chromaticity of $\xi \approx 15$ was used when the beam with bunch spacing of $25 \mathrm{~ns}$ injected into the LHC, which allowed beam to survive for scrubbing.

The negative effect of the large chromaticity has been proved acceptable for the goal of beam scrubbing, despite that the beam quality is degraded.

The second problem in the injection scrubbing is related to the longer bunch and larger beam momentum spread, which is shown in Table 3, where the bunch length and beam momentum spread at the store are also shown for comparison. 
The Injection 1 is with the RF frequency $28.1 \mathrm{MHz}$ and the $\mathrm{RF}$ voltage $300 \mathrm{kV}$, which is comparable to RHIC proton operations. In Injection 1, the rms bunch length is $\sigma_{t}=2 n s$, which is 8 times of that at the store. This much longer bunch length is expected to have much weaker electron cloud, and therefore, with less effective beam scrubbing.

\begin{tabular}{|c|c|c|c|c|}
\hline & Inject. 1 & Inject. 2 & Inject. 3 & Store \\
\hline Relativistic gamma, $\gamma$ & 25.4 & 25.4 & 25.4 & 271 \\
\hline RF frequency, $f_{r f}, M H z$ & 28.1 & & & 394.1 \\
\hline RF Voltage, $V_{r f}, M V$ & 0.3 & & & 13.8 \\
\hline rms bunch length in time, $\sigma_{t}, n s$ & 2.0 & 1.0 & 0.5 & 0.25 \\
\hline rms bunch length, $\sigma_{s}, c m$ & 60 & 30 & 15 & 8.0 \\
\hline rms momentum spread, $(d p / p)_{r m s}, 10^{-4}$ & 13.5 & 27 & 54 & 6.5 \\
\hline
\end{tabular}

Table 3. Beam parameters at injection and store, with the design longitudinal emittance of $0.8 \mathrm{eVs}$. The injection 1 is close to what we have now. Injection 2 and Injection 3 are listed due to the beam scrubbing considerations.

For better scrubbing, Injections 2 and 3, with shorter bunches, are listed, the harmonic and $\mathrm{RF}$ voltages are not specified, but the associated beam momentum spread can be calculated with the given longitudinal emittance $A_{\ell}$, as below,

$$
A_{\ell}=4 \pi \beta \gamma E_{0} \sigma_{t}(d p / p)_{r m s}
$$

In Table 4, the heat load simulated for dipoles at the eRHIC injection is presented with different bunch lengths shown in Table 3.

\begin{tabular}{|c|c|c|c|c|c|}
\hline & $\sigma_{t}$ & $d n_{e} / d s$ & Energy Impact & $d P / d s$ & $\langle E\rangle$ \\
\hline & $n s$ & $10^{9} / m$ & $10^{11} \mathrm{eV}$ & $W / m$ & $e V$ \\
\hline \multicolumn{7}{|c|}{ Injection, $\mathrm{B}=0.32 \mathrm{~T}$} \\
\hline Injection 1 & 2.0 & 3.3 & 2.1 & 0.96 & 63 \\
\hline Injection 2 & 1.0 & 3.9 & 3.9 & 1.78 & 100 \\
\hline Injection 3 & 0.5 & 4.2 & 5.3 & 2.42 & 126 \\
\hline \multicolumn{7}{|c|}{ Store, $\mathrm{B}=3.5 \mathrm{~T}$} \\
\hline Store & 0.25 & 4.1 & 7.0 & 3.20 & 170 \\
\hline
\end{tabular}

Table 4. Beam scrubbing at the injection for different bunch lengths. The one at the store is presented for comparison. All are with the $\mathrm{SEY} \delta_{\max }=2.1$.

For Injection 1, even with all 330 bunches injected, the heat load is $0.96 \mathrm{~W} / \mathrm{m}$, and the scrubbing cannot proceed any further by adding more bunches. With this respect, Injections 2 and 3 have some rooms to go for further scrubbing. 
On the other hand, even for Injection 1 , the beam momentum spread $(d p / p)_{r m s}=13.5 \times 10^{-4}$ is quite large, which is likely causing the reduced beam lifetime. With the beams in Injections 2 and 3, the even larger beam momentum spread will affect more on the beam lifetime.

The solution for the reduced beam lifetime is to use frequent beam injections, and fortunately, this is not difficult to do at the injection.

The third problem for the injection scrubbing is that the scrubbed area on the chamber surface is not well matched to the one at the beam store for physics runs.

In Figure 5, the beam scrubbed area is shown for different bunch lengths.

The scrubbed area with $\sigma_{t}=2 n s$ is not matched well with the physics runs. Consider that this option also has low scrubbing efficiency, therefore, it is not preferable.

The scrubbed area with $\sigma_{t}=1 n s$ and $\sigma_{t}=0.5 n s$ covers that of the physics runs, whereas the latter is the better choice.

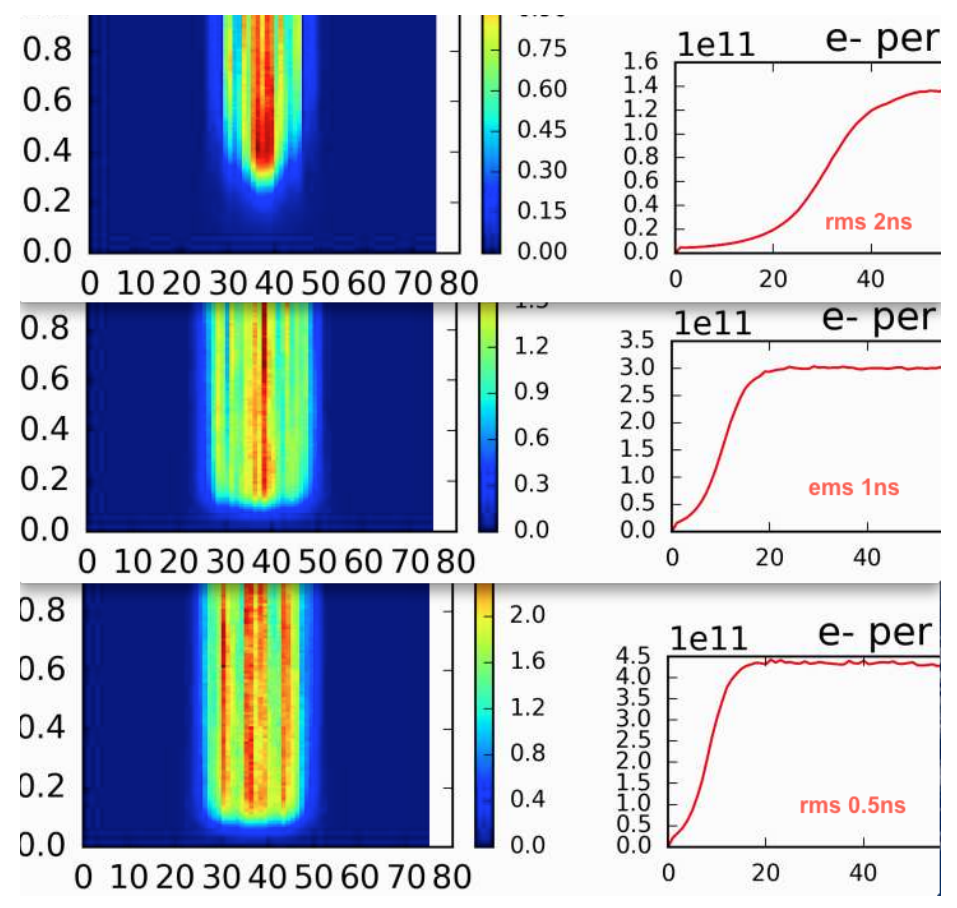

Figure 5: Beam scrubbing at injection with different bunch lengths. From top to button are with $\sigma_{t}=2 n s, \sigma_{t}=1 n s$ and $\sigma_{t}=0.5 n s$. The scrubbed area is not well matched with the area relevant to physics runs, which has $\sigma_{t}=0.25 \mathrm{~ns}$. The one with $\sigma_{t}=0.5 \mathrm{~ns}$ is the best choice for this concern.

With the problems in the injection scrubbing, it is not straightforward to plan for specific details. On the other hand, all the problems can be mitigated in some ways.

1. The beam instability can be mitigated by using large chromaticity, as LHC has demonstrated in the operations.

2. The possible short beam lifetime can be mitigated by frequent injections. 
3. The needed scrubbed area is in general covered by the beams with different bunch lengths and intensities. Therefore, the injection scrubbing may be performed with various beams of different bunch lengths and intensities.

Once the SEY is reduced to around $\delta_{\max }=1.5$, the beam can be ramped to full energy, then a physics scrubbing can be performed, for that, the scrubbing plan shown in Figure 4 can be applied.

\section{References}

[1] M. A. Furman, The Electron-Cloud Effect in the Arcs of the LHC, CERN LHC Project Report 180, 1998.

[2] R. Cimino, I. R. Collins, M. A. Furman, M. Pivi, F. Ruggiero, G. Rumolo, and F. Zimmermann, Can Low-Energy Electrons Affect High-Energy Physics Accelerators? PRL, 93, 014801, 2004.

[3] V. Baglin, I. Collins, B. Henrist, N. Hilleret, and G. Vorlaufer, A Summary of Main Experimental Results Concerning the Secondary Electron Emission of Copper, LHC Project Report 472, 2002.

[4] R. Cimino, M. Commisso, D. R. Grosso, T. Demma, V. Baglin, R. Flammini, and R. Larciprete, Nature of the decrease of the secondary-electron yield by electron bombardment and its energy dependence, PRL, 109, 064801, 2012.

[5] G. Iadarola, L. Mether, and G. Rumolo, Are the electron clouds clearing? LHC Performance Workshop, Jan. 23-27, 2017.

[6] C. Montag et. al., eRHIC Ring-Ring Design, BNL, 2016.

[7] G. Iadarola, Electron Cloud studies for CERN particle accelerators and simulation code development, CERN, Thesis, 2014.

[8] F. Zimmermann, Review of single bunch instabilities driven by an electron cloud, PRST AB, 7, 124801, 2004.

[9] K. Li and G. Rumolo, Review of Beam Instabilities in the Presence of Electron Clouds in the LHC, p.760, IPAC11, 2011 